\title{
Femtosecond time-resolved dynamical Franz-Keldysh effect
}

\author{
T. Otobe ${ }^{1}$, Y. Shinohara ${ }^{2}$, S. A. Sato ${ }^{3}$, and K. Yabana ${ }^{1,3,4}$ \\ ${ }^{1}$ Kansai Photon Science Institute, Japan Atomic Energy Agency, Kizugawa, Kyoto 619-0615, Japan \\ ${ }^{2}$ Max-Planck Institut für Mikrostrukturphysik, Weinberg 2, D-06120, Halle, Germany \\ ${ }^{3}$ Graduate School of Pure and Applied Sciences, University of Tsukuba, Tsukuba 305-8571, Japan \\ ${ }^{4}$ Center for Computational Sciences, University of Tsukuba, Tsukuba 305-8577, Japan
}

\begin{abstract}
We theoretically investigate the dynamical Franz-Keldysh effect in femtosecond time resolution, that is, the time-dependent modulation of a dielectric function at around the band gap under an irradiation of an intense laser field. We develop a pump-probe formalism in two distinct approaches: first-principles simulation based on real-time time-dependent density functional theory and analytic consideration of a simple two-band model. We find that, while time-average modulation can be reasonably described by the static Franz-Keldysh theory, a remarkable phase shift is found to appear between the dielectric response and the applied electric field.
\end{abstract}

\section{INTRODUCTION}

In last two decades, intense coherent light of different characteristics has become available owing to advances in laser sciences and technologies. Ultrashort laser pulses can be as short as a few tens of attosecond, forming a new field of attosecond science [1]. Intense laser pulses of mid-infrared (MIR) or $\mathrm{THz}$ frequencies have also become available recently [2, 3]. Employing these extreme sources of coherent light, it is possible to investigate the optical response of materials in real time with a resolution much less than an optical cycle [1, 4, 8].

The dielectric function $\varepsilon(\omega)$ is the most fundamental quantity characterizing the optical properties of matter. Modulation of the dielectric function $\varepsilon(\omega)$ in the presence of electromagnetic fields has been a subject of investigation for many years. The change under a static electric field is known as the Franz-Keldysh effect (FKE) [9[16], and that under an alternating electric field is known as the dynamical FKE (DFKE) [17 22]. An important parameter which distinguishes DFKE from the static FKE is the adiabaticity parameter $\gamma=U_{p} / \Omega$, where $U_{p}=e^{2} E^{2} / 4 \mu \Omega^{2}$ is the ponderomotive energy, and $\Omega$ is the frequency of the field, $\mu$ is the reduced mass of the electron, and $E$ is the electic field [19]. A multi-photon picture applies for $\gamma<<1$, and a static FKE picture is appropriate for $\gamma>>1$. Laser pulses having $\gamma \sim 1$ is an intriguing regime where novel and unobvious DFKE phenomena are expected.

In previous investigations of DFKE, the main focus was on the modulation of the optical response averaged over times much longer than the optical cycle, examining the time-averaged fine structure [18] and shifts in excitation structures [19]. In the present paper, we examine DFKE in time domain, with a resolution much less than the cycle of the applied optical field. The DFKE response in subfemtosecond time resolution is very relevant to ultrafast optical switching in the teraherz or even petahertz $\left(10^{15}\right.$ hertz $)$ domain [5, 7]. A first experimental report on the DFKE with a femtosecond time resolution has recently been given by Novelli et al. 7] for GaAs, employing an intense pump pulse of $\mathrm{THz}$ fre- quency. They observed an interesting time shift between the pump pulse and the modulation of dielectric function, but the mechanism of the observed time profile was not understood. To uncover the physics of time-resolved DFKE, we develop a pump-probe formalism in two different theoretical approaches: first-principles numerical simulations based on time-dependent density functional theory (TDDFT 25] ) and analytic investigation for a two-band model. Combining two approaches, we can understand not only the strength of the modulation but the phase with respect to the pump field as well.

The organization of the present article is as follows. In section II, we present formalism and results of our first-principles calculations for the time-resolved DFKE. In section III, we develop an analytical formalism for the time-resolved DFKE employing a parabolic two-band model. In section IV, a summary will be presented.

\section{FIRST-PRINCIPLES PUMP-PROBE CALCULATION}

\section{A. Formalism}

In real-time TDDFT, we describe electron dynamics in a unit cell of a crystalline solid under a spatially-uniform electric field $\vec{E}(t)$. The method has been applied for calculations of linear optical responses 26] and nonlinear electronic excitations by intense laser pulses [28 34]. Treating the field by a vector potential

$$
\vec{A}(t)=-c \int^{t} d t^{\prime} \vec{E}\left(t^{\prime}\right)
$$

the electron dynamics in the unit cell of solid is described by the following time-dependent Kohn-Sham (TDKS) equation [26] :

$$
\begin{aligned}
& i \frac{\partial}{\partial t} \psi_{i}(\vec{r}, t)=\left[\frac{1}{2 m_{e}}\left(\vec{p}+\frac{e}{c} \vec{A}(t)\right)^{2}\right. \\
& \left.+V_{i o n}(\vec{r})+V_{H}(\vec{r}, t)+V_{x c}(\vec{r}, t)\right] \psi_{i}(\vec{r}, t),
\end{aligned}
$$

where $m_{e}$ is the electron mass, $V_{i o n}$ is the electron-ion potential for which we use a norm-conserving pseudpo- 
tential [35, [36], and $V_{H}(\vec{r}, t)$ and $V_{x c}(\vec{r}, t)$ are electronelectron Hartree and exchange-correlation potentials, respectively. For the exchange-correlation potential, we employ an adiabatic local density approximation, using the same functional form of the potential for both ground state and time evolution calculations [37]. Since the Kohn-Sham Hamiltonian in Eq. (2) has the lattice periodicity at each time, we may introduce time-dependent Bloch wave function, $\psi_{i}(\vec{r}, t)=e^{i \vec{k} \cdot \vec{r}} u_{n \vec{k}}(\vec{r}, t)$. In practice, we calculate the time evolution of the Bloch wave functions.

We calculate electron dynamics in diamond, using a cubic unit cell containing eight carbon atoms. The TDKS equation is solved in real time and real space. The realspace grids of $22^{3}$ is used for the unit cell, and $32^{3}$ grids for the $k$-points. The Taylor expansion method is used for the time evolution 38 with a time step of $\Delta t=0.02$ in atomic unit. The number of time steps is typically 70,000 . An important output of the calculation is the average electric current density as a function of time, $\vec{J}(t)$. It is given by

$$
\vec{J}(t)=-\frac{e}{m_{e} V} \int_{V} d \vec{r} \sum_{i} \operatorname{Re} \psi_{i}^{*}\left(\vec{p}+\frac{e}{c} \vec{A}(t)\right) \psi_{i}+J_{N L}(t),
$$

where $V$ is a volume of the unit cell. $\vec{J}_{N L}(t)$ is the current caused by non-locality of the pseudopotential.

It should be mentioned that $\vec{A}(t)$ in the TDKS equation (2) is the vector potential in the medium and not that of the incident pulse in the vacuum. As we discussed in Ref. [39], the relation between the two depends on the macroscopic shape of the materials as well as the direction of the polarization. Exchange-correlation effects may also appear in the vector potential $\vec{A}(t)$ in timedependent current density functional theory [40], which we ignore for simplicity.

To examine the DFKE, we carry out simulations solving the TDKS equation (2) including both pump and probe electric fields in the vector potential $\vec{A}(t)$ 33]. We assume that both pump and probe electric fields are linearly polarized and are orthogonal to each other. We denote the pump electric field as $E_{P}(t)$ and the probe electric field as $E_{p}(t)$. The probe electric field is assumed to be weak enough to be treated by the linear response theory. We denote the electric current caused by the probe field as $J_{p}(t)$, which is assumed to be parallel to the direction of the probe electric field. They are related by the time-domain conductivity $\sigma\left(t, t^{\prime}\right)$ as

$$
J_{p}(t)=\int_{-\infty}^{t} d t^{\prime} \sigma\left(t, t^{\prime}\right) E_{p}\left(t^{\prime}\right)
$$

We note that the conductivity $\sigma\left(t, t^{\prime}\right)$ depends on both times $t$ and $t^{\prime}$ rather than just time difference $t-t^{\prime}$ due to the presence of the pump pulse.

We derive a frequency-dependent conductivity at time $T$ from the conductivity $\sigma\left(t, t^{\prime}\right)$. We first note that, in the absence of the pump electric field, the frequencydependent conductivity $\tilde{\sigma}(\omega)$ is related to the Fourier transforms of the electric field and the induced current as $\tilde{\sigma}(\omega)=\int d t e^{i \omega t} J_{p}(t) / \int d t e^{i \omega t} E_{p}(t)$, where we may use any time profile for the probe electric field, $E_{p}(t)$. We employ this relation in the presence of the strong pump field. To introduce the time $T_{p}$ to explore the response, a pulsed electric field is employed for $E_{p}(t)$ whose envelope shows a maximum at $t=T_{p}$. Namely, we use the following relation to define the time-resolved, frequencydependent conductivity $\sigma\left(T_{p}, \omega\right)$,

$$
\tilde{\sigma}\left(T_{p}, \omega\right)=\frac{\int d t e^{i \omega t} J_{p}(t)}{\int d t e^{i \omega t} E_{p}(t)} .
$$

Thus, in this consideration, the probe electric field has a dual role, to specify the time $T_{p}$ at which we explore the response of the medium and to distort the medium to examine responses of the system. We consider that this dual role of the probe pulse will be adopted to measure the transient dielectric functions in most experiments.

\section{B. Numerical results}

In the calculations below, we use the following electric fields. The pump field is of the form $E_{P}(t)=$ $E_{0, P} f_{P}(t) \sin \Omega t$ with the central angular frequency $\Omega$ set to $\Omega=0.4 \mathrm{eV}$; its direction is along the [001] axis. The field is turned on adiabatically described by the function $f_{P}(t)$,

$$
f_{P}(t)= \begin{cases}\sin ^{2}\left(\frac{\pi}{2 T_{P}} t\right) & \left(0<t<T_{P}\right) \\ 1 & \left(t \geq T_{P}\right)\end{cases}
$$

where $T_{P}$ is set to $10 \mathrm{fs}$. The probe field is of the form

$$
E_{p}(t)=E_{0, p} \sin \left(\omega_{p} t\right) \exp \left(-\frac{\left(t-T_{p}\right)^{2}}{\eta^{2}}\right),
$$

oriented in the [100] direction. The average frequency $\omega_{p}$ is set to $5.6 \mathrm{eV}$, which is equal to the calculated band gap energy of diamond in LDA. The field strength is set to $E_{0, p}=2.7 \times 10^{-3} \mathrm{MV} / \mathrm{cm}$, which is small enough to probe the linear response of the medium. The pulse duration $\eta$ is set to $\eta=0.7$ fs. With such a short duration, we may scan the spectrum of broad frequency region around the band gap from a single pump-probe calculation.

The frequency-dependent conductivity is calculated using Eq. (5) with a slight modification,

$$
\tilde{\sigma}\left(T_{p}, \omega\right)=\frac{\int d t e^{i \omega t} g\left(t-T_{p}\right) J_{p}(t)}{\int d t e^{i \omega t} E_{p}(t)},
$$

where we introduced a filter function, $g(t)$, [41] to suppress spurious oscillations which arise from a finite time period of the simulation. 

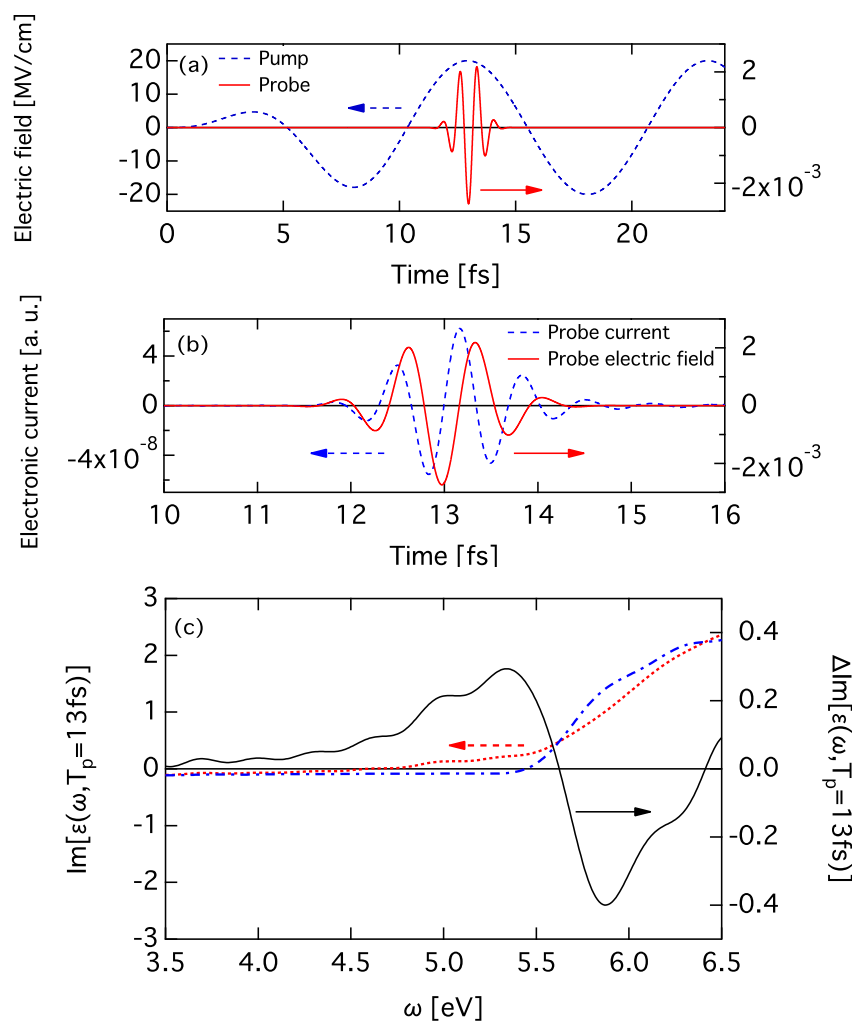

FIG. 1. (a) Pump (blue-dashed line) and probe (red-solid line) electric fields are shown. (b) The electronic current (blue-dashed line) induced by the probe electric field (redsolid line). (c) The imaginary part of the dielectric function in the presence of the pump field, $\operatorname{Im}\left[\varepsilon\left(\omega, T_{p}=13 \mathrm{fs}\right)\right]$ (reddashed line), and in the absence of the pump field, $\operatorname{Im}[\varepsilon(\omega)]$ (blue-dash-dot line). Black-solid line shows the difference, $\operatorname{Im}\left[\varepsilon\left(\omega, T_{p}=13 f s\right)\right]-\operatorname{Im}[\varepsilon(\omega)]$.

Figure 1 shows an example of our pump-probe calculations. In (a), the electric fields of the pump field $E_{P}(t)$ and the probe pulse $E_{p}(t)$ are presented: The bluedashed line shows the electric fields of the pump field $E_{P}(t)$, and the red-solid line shows the electric field of the probe pulse $E_{p}(t)$. The magnitude of the pump electric field, $E_{0, P}$, is set to $20 \mathrm{MV} / \mathrm{cm}$. The probe pulse is applied at a time when the magnitude of the pump field is maximum. The electric current induced by the probe pulse, $J_{p}(t)$, is shown by the blue-dashed line in (b). The conductivity is calculated from the probe current using Eq. (8), and then converted to the dielectric function using a formula

$$
\epsilon(\omega)=1+4 \pi i \frac{\sigma(\omega)}{\omega}
$$

which is valid in the presence of the strong pump field. The imaginary part of the dielectric function, $\operatorname{Im}\left[\varepsilon\left(\omega, T_{p}=13 f s\right)\right]$, is shown in (c). The red-dotted and blue dot-dashed lines present the dielectric function with and without the pump field, respectively. A change of the imaginary part of the dielectric function,
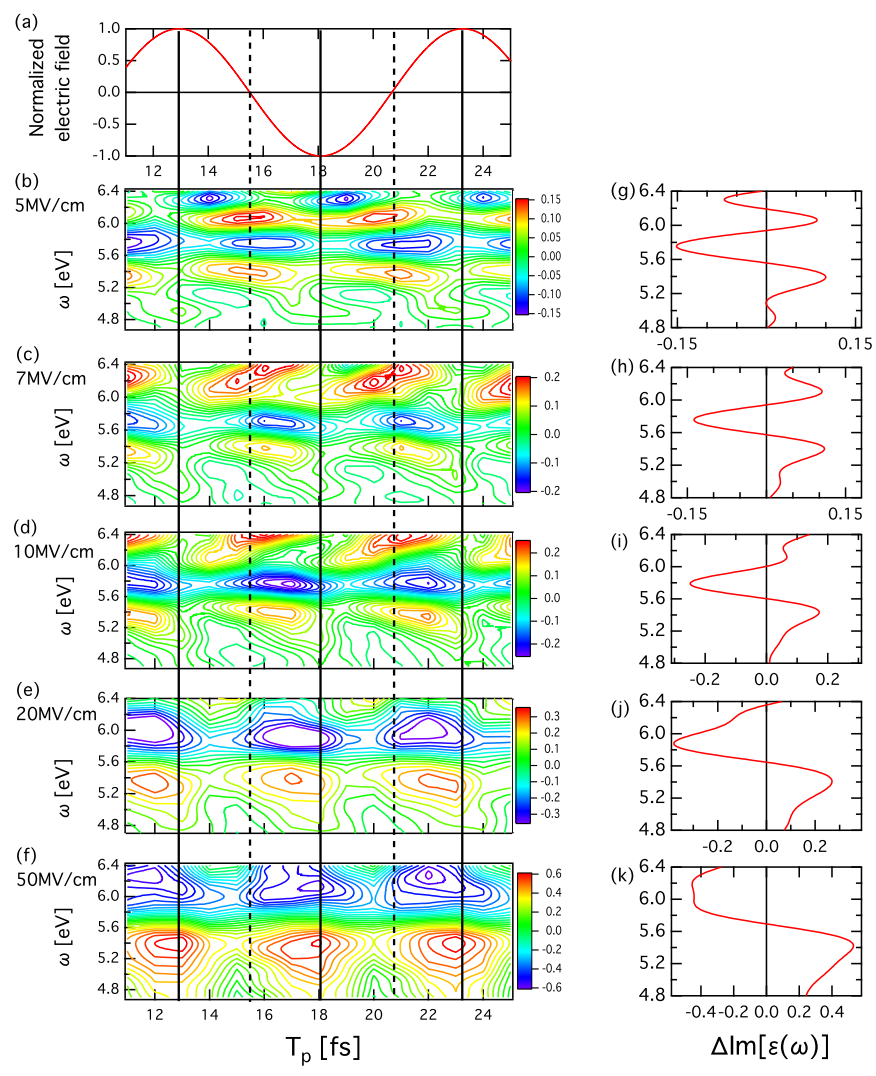

FIG. 2. Contour plots (left) and their time averages (right) of $\Delta \operatorname{Im}\left[\varepsilon\left(\omega, T_{p}\right)\right]$ under the MIR pump field of the intensity of $(\mathrm{b}, \mathrm{g}) 5,(\mathrm{c}, \mathrm{h}) 7,(\mathrm{~d}, \mathrm{i}) 10,(\mathrm{e}, \mathrm{j}) 20$, and $(\mathrm{f}, \mathrm{k}) 50 \mathrm{MV} / \mathrm{cm}$. The time dependence of the pump electric field is shown in (a). In panels (a)-(f), the vertical solid (dashed) lines indicate the time of maximum (zero) of the pump electric field.

$\Delta \operatorname{Im}\left[\varepsilon\left(\Omega, T_{p}=13 f s\right)\right]=\operatorname{Im}\left[\varepsilon\left(\Omega, T_{p}=13 f s\right)\right]-\operatorname{Im}[\varepsilon(\omega)]$, is shown by the black-solid line. It indicates modulations of an exponential tail below and an oscillation above the band gap $(\omega=5.5 \mathrm{eV})$.

Figure 2 shows changes of the imaginary part of the dielectric function caused by the strong pump field, $\Delta \operatorname{Im}\left[\varepsilon\left(\omega, T_{p}\right)\right]=\operatorname{Im}\left[\varepsilon\left(\omega, T_{p}\right)\right]-\operatorname{Im}[\varepsilon(\omega)] . \quad \operatorname{In}(\mathrm{a})$, time profile of the pump electric field is shown. In (b) - (f), changes of imaginary part of the dielectric function are shown in contour plots for four intensities. Horizontal axis is the time $T_{p}$ and the vertical axis is the frequency $\omega$. In (g)-(k), modulations averaged over time are shown as a function of frequency.

We first look at the case of strongest pump electric field, $E_{0, P}=50 \mathrm{MV} / \mathrm{cm}$,shown in (f) and (k). We find that an increase of the absorption below and a decrease above the band gap are seen when the magnitude of the pump electric field is close to the maximum. The modulation is small when the electric field is close to zero. This fact indicates the static FKE appears instantaneously following the change of the pump field. We confirmed the modulation is well fitted by the static FKE formula if we assume the effective mass of $\mu=0.25 \mathrm{~m}$. With this 
value of the effective mass, the adiabatic parameter $\gamma$ is 29.5, much larger than unity, which is consistent with the appearance of the static FKE.

As the field intensity decreases, we find changes in two aspects, one along the frequency axis and the other along the time axis. Above the band gap, we find an oscillatory behavior in the frequency direction. This is clearly seen in the time-average behaviors shown in $(\mathrm{g})-(\mathrm{k})$. For example, at the electric field of $10 \mathrm{MV} / \mathrm{cm}$, shown in (d) and (i), the modulation is negative between $5.6 \mathrm{eV}$ (band gap) to $6.0 \mathrm{eV}$. It then becomes positive above $6.0 \mathrm{eV}$.

Along the time axis, we can see a striking change in the phase between the modulation and the pump electric field. At the strongest electric field (f), the modulation and the pump electric field is in phase, as mentioned above. As the intensity of the pump field decreases, a phase shift forward in time is seen. The amount of the phase difference increases as the magnitude of the pump field decreases, as seen from (b) to (f). At the lowest intensity (b), the modulation signals appear at times when the pump field is close to zero. The parameter $\gamma$ for these intensities is (b) 0.29 , (c) 0.58, (d) 1.19, and (e) 4.76. Thus the phase change becomes appreciable when the $\gamma$ value is around and below unity.

\section{ANALYTIC CONSIDERATION}

\section{A. General formula for the conductivity in the presence of a strong field}

We next develop an analytic consideration to understand behaviors of the DFKE signals seen in the numerical simulation. In the following developments, we consider a simplified description: electron dynamics in the presence of pump and probe fields is assumed to be described by a time-dependent Schrödinger equation for a single electron,

$$
i \frac{\partial}{\partial t} \psi_{i}(\vec{r}, t)=\left[\frac{1}{2 m_{e}}\left(\vec{p}+\frac{e}{c} \vec{A}(t)\right)^{2}+V(\vec{r})\right] \psi_{i}(\vec{r}, t)
$$

where $V(\vec{r})$ is a time-independent, lattice periodic potential. We thus ignore the time-dependence of the Kohn-Sham Hamiltonian of Eq. (2), except for the vector potential. We express the solution of this equation using time-dependent Bloch function $v_{n \vec{k}}(\vec{r}, t)$ as $\psi_{i}(\vec{r}, t)=e^{i \vec{k} \vec{r}} v_{n \vec{k}}(\vec{r}, t)$.

We further assume that, in the presence of the pump field described by a vector potential $\vec{A}_{P}(t)$, the solution of Eq. (10) is well approximated by the so-called Houston function [17, 42]. Using static Bloch orbitals $u_{n \vec{k}}(\vec{r})$ and orbital energies $\epsilon_{n \vec{k}}$ which satisfy

$$
\left[\frac{1}{2 m_{e}}(\vec{p}+\vec{k})^{2}+V(\vec{r})\right] u_{n \vec{k}}(\vec{r})=\epsilon_{n \vec{k}} u_{n \vec{k}}(\vec{r})
$$

the Houston function is expressed as

$$
w_{n \vec{k}}(\vec{r}, t)=u_{n \vec{k}_{P}(t)}(\vec{r}) \exp \left[-i \int^{t} \epsilon_{n \vec{k}_{P}\left(t^{\prime}\right)} d t^{\prime}\right],
$$

where $\vec{k}_{P}(t)$ is defined by $\vec{k}_{P}(t)=\vec{k}+e \vec{A}_{P}(t) / c$.

We then consider the solution of Eq. (10) in the presence of both pump and probe fields, $\vec{A}(t)=\vec{A}_{P}(t)+\vec{A}_{p}(t)$. We express the time-dependent Bloch function as

$$
v_{n \vec{k}}(\vec{r}, t)=w_{n \vec{k}}(\vec{r}, t)+\sum_{m} C_{n m}^{\vec{k}}(t) w_{m \vec{k}}(\vec{r}, t),
$$

where the coefficients $C_{n m}^{\vec{k}}(t)$ are determined by the standard procedure in the time-dependent perturbation theory. We have

$$
\begin{aligned}
C_{n m}^{\vec{k}}(t)= & -\frac{i e}{m_{e} c} \int_{-\infty}^{t} d t^{\prime} \vec{P}_{m n}^{\vec{k}}\left(t^{\prime}\right) \vec{A}_{p}\left(t^{\prime}\right) \\
& -\delta_{m n} \frac{i e}{m_{e} c} \int_{-\infty}^{t} d t^{\prime} \vec{k}_{P}\left(t^{\prime}\right) \vec{A}_{p}\left(t^{\prime}\right)
\end{aligned}
$$

Here we have introduced matrix elements of momentum operator,

$$
\begin{aligned}
& \vec{P}_{m n}^{\vec{k}}(t)=\int_{V} d \vec{r} w_{m \vec{k}}^{*}(\vec{r}, t) \vec{p} w_{n \vec{k}}(\vec{r}, t) \\
= & (\vec{p})_{n n^{\prime} \vec{k}_{P}(t)} \exp \left[-i \int_{-\infty}^{t} d t^{\prime}\left(\epsilon_{n \vec{k}_{P}\left(t^{\prime}\right)}-\epsilon_{n^{\prime} \vec{k}_{P}\left(t^{\prime}\right)}\right)\right],
\end{aligned}
$$

where

$$
(\vec{p})_{n n^{\prime} \vec{k}}=\int_{V} d \vec{r} u_{n \vec{k}}^{*}(\vec{r}) \vec{p} u_{n^{\prime} \vec{k}}(\vec{r})
$$

is the matrix element in the static basis.

The electric current density averaged over the unit cell is given by

$$
\begin{aligned}
\vec{J}(t)= & -\frac{e}{m_{e} V} \int_{V} d \vec{r} \\
& \sum_{n \vec{k}} \operatorname{Re}\left\{v_{n \vec{k}}^{*}\left(\vec{p}+\vec{k}_{P}(t)+\frac{e}{c} \vec{A}_{p}(t)\right) v_{n \vec{k}}\right\} .
\end{aligned}
$$

This may be decomposed into pump and probe contributions, $\vec{J}(t)=\vec{J}_{P}(t)+\vec{J}_{p}(t)$. The two components are

$$
\begin{gathered}
\vec{J}_{P}(t)=-\frac{e}{m_{e} V} \sum_{n \vec{k}} \int_{V} d \vec{r} \operatorname{Re}\left\{w_{n \vec{k}}^{*}\left(\vec{p}+\vec{k}_{P}(t)\right) w_{n \vec{k}}\right\}, \\
\vec{J}_{p}(t)=-\frac{e^{2}}{m_{e} c} n_{e} \vec{A}_{p}(t)+\frac{e^{2}}{m_{e}^{2} c V} \int_{-\infty}^{t} d t^{\prime} \\
\sum_{n \neq n^{\prime}, \vec{k}} \operatorname{Im}\left\{\vec{P}_{n n^{\prime}} \vec{k}(t)\left(\vec{P}_{n^{\prime} n}^{\vec{k}}\left(t^{\prime}\right) \cdot \vec{A}_{p}\left(t^{\prime}\right)\right)\right\}
\end{gathered}
$$

where $n_{e}$ is the average density of valence electrons. 
Equation (19) is a linear relation between the probe field and the induced electric current density. As we described in Eq. (4), we introduce a conductivity function $\sigma_{\alpha \beta}\left(t, t^{\prime}\right)$ that relates the probe field and the induced electric current density,

$$
J_{\alpha}^{p}(t)=\sum_{\beta} \int_{-\infty}^{\infty} d t^{\prime} \sigma_{\alpha \beta}\left(t, t^{\prime}\right) E_{\beta}^{p}\left(t^{\prime}\right),
$$

where $\alpha, \beta$ are the Cartesian indices of the current and the probe field, and $J_{\alpha}^{p}$ and $E_{\alpha}^{p}$ indicate $\alpha$-components of probe electric current and probe electric field, respectively. The conductivity function for $t>t^{\prime}$ is given by

$$
\begin{aligned}
& \sigma_{\alpha \beta}\left(t, t^{\prime}\right)=\frac{e^{2}}{m_{e}} n_{e} \delta_{\alpha \beta} \\
&- \frac{e^{2}}{m_{e}^{2} V} \int_{t^{\prime}}^{t} d t^{\prime \prime} \sum_{n \neq n^{\prime}, \vec{k}} \operatorname{Im}\left[\left(p_{\alpha}\right)_{n n^{\prime} \vec{k}_{P}(t)}\left(p_{\beta}\right)_{n^{\prime} n \vec{k}_{P}\left(t^{\prime \prime}\right)}\right. \\
&\left.\times \exp \left[-i \int_{t^{\prime \prime}}^{t} d \tau\left\{\epsilon_{n^{\prime} \vec{k}_{P}(\tau)}-\epsilon_{n \vec{k}_{P}(\tau)}\right\}\right]\right] .
\end{aligned}
$$

This is our general expression for the conductivity in the presence of a strong electric field, when the system is described by the time-dependent Schrödinger equation (10).

The polarization induced by the probe field is given as the integral of the current over time. We may introduce the linear susceptibility $\chi_{\alpha \beta}\left(t, t^{\prime}\right)$ by

$$
P_{\alpha}^{p}(t)=\int_{-\infty}^{t} d t^{\prime} J_{p}\left(t^{\prime}\right)=\sum_{\beta} \int_{-\infty}^{t} d t^{\prime} \chi_{\alpha \beta}\left(t, t^{\prime}\right) E_{\beta}^{p}\left(t^{\prime}\right) .
$$

Therefore, the linear susceptibility and the conductivity are related by

$$
\chi_{\alpha \beta}\left(t, t^{\prime}\right)=\int_{-\infty}^{t} d t^{\prime \prime} \sigma_{\alpha \beta}\left(t^{\prime \prime}, t^{\prime}\right) .
$$

\section{B. Time-resolved, frequency-dependent conductivity}

As we described in Sec. IIA we introduce a timeresolved, frequency-dependent conductivity by Eq. (5) using a probe electric field $E_{\beta}^{p}(t)$ which has a sharp peak at $t=T_{p}$

$$
\tilde{\sigma}_{\alpha \beta}\left(T_{p}, \omega\right)=\frac{\tilde{J}_{\beta}^{p}(\omega)}{\tilde{E}_{\alpha}^{p}(\omega)},
$$

where $\tilde{J}_{\alpha}^{p}(\omega)$ and $\tilde{E}_{\alpha}^{p}(\omega)$ are Fourier transforms of $J_{\alpha}^{p}(t)$ and $E_{\alpha}^{p}(t)$, respectively. As a probe field, we first consider an impulsive probe field at $t=T_{p}, E_{\beta}^{p}(t)=k \delta\left(t-T_{p}\right)$, where $k$ specifies the strength of the probe pulse. With this simple choice of the probe field, we have the following result for the time-resolved conductivity,

$$
\tilde{\sigma}_{\alpha \beta}^{I}\left(T_{p}, \omega\right)=\int d s e^{i \omega s} \sigma_{\alpha \beta}\left(T_{p}+s, T_{p}\right),
$$

where the superscript $I$ of $\tilde{\sigma}_{\alpha \beta}^{I}$ indicates that the impulsive probe field is used. As a more general probe pulse, we consider

$$
E_{\beta}^{p}(t)=f_{p}\left(t-T_{p}\right) e^{-i \omega_{p}\left(t-T_{p}\right)}
$$

where $\omega_{p}$ indicates an average frequency of the probe pulse and $f_{p}(t)$ is a real envelope function having a peak at $t=0$. We assume that it is an even function, $f_{p}(t)=f_{p}(-t)$, so that the Fourier transform, $\tilde{f}_{p}(\omega)=\int d t e^{i \omega t} f_{p}(t)$ is real. With this choice of the probe pulse and using Eq. (24), we have

$$
\tilde{\sigma}_{\alpha \beta}\left(T_{p}, \omega\right)=\frac{\int d s f_{p}(s) \tilde{\sigma}_{\alpha \beta}^{I}\left(T_{p}+s, \omega\right) e^{i\left(\omega-\omega_{p}\right) s}}{\int d s f_{p}(s) e^{i\left(\omega-\omega_{p}\right) s}} .
$$

This expression indicates that the time-resolved conductivity does not depend much on details of the shape of the probe pulse if we use a short enough probe pulse $f_{p}$ so that $\tilde{\sigma}_{\alpha \beta}^{I}\left(T_{p}+s, \omega\right)$ does not change much in the duration of the probe pulse.

We note that the real part of the time-resolved conductivity $\operatorname{Re} \tilde{\sigma}_{\alpha \alpha}\left(T_{p}, \omega\right)$ is related to the energy transfer from the probe electric field to electrons, as the ordinary conductivity does. To show it, we consider the work done by the probe electric field to electrons which is given by

$$
W=\int d t \vec{E}_{p}(t) \vec{J}_{p}(t)
$$

This can be written as

$$
\begin{aligned}
W & =\sum_{\alpha} \int d t E_{\alpha}^{p}(t) J_{\alpha}^{p}(t) \\
& =\sum_{\alpha} \frac{1}{2 \pi} \int d \omega \tilde{E}_{\alpha}^{p *}(\omega) \tilde{J}_{\alpha}^{p}(\omega) \\
& =\sum_{\alpha} \frac{1}{2 \pi} \int d \omega\left|\tilde{E}_{\alpha}^{p}(\omega)\right|^{2} \frac{J_{\alpha}^{p}(\omega)}{E_{\alpha}^{p}(\omega)} \\
& =\sum_{\alpha} \frac{1}{2 \pi} \int d \omega\left|\tilde{E}_{\alpha}^{p}(\omega)\right|^{2} \tilde{\sigma}_{\alpha \alpha}\left(T_{p}, \omega\right),
\end{aligned}
$$

where we used the definition for the $\tilde{\sigma}_{\alpha \beta}\left(T_{p}, \omega\right)$ given by Eq. (27). Since the physical electric field, $E_{\alpha}^{p}(t)$, and the physical induced current, $J_{\alpha}^{p}(t)$, are real quantities, we have

$$
\tilde{\sigma}_{\alpha \alpha}^{*}\left(T_{p}, \omega\right)=\tilde{\sigma}_{\alpha \alpha}\left(T_{p},-\omega\right) .
$$

Using this relation, we have

$$
\begin{aligned}
W & =\sum_{\alpha} \frac{1}{2 \pi} \int_{-\infty}^{\infty} d \omega\left|\tilde{E}_{\alpha}^{p}(\omega)\right|^{2} \tilde{\sigma}_{\alpha \alpha}\left(T_{p}, \omega\right) \\
& =\sum_{\alpha} \frac{1}{\pi} \int_{0}^{\infty} d \omega\left|\tilde{E}_{\alpha}^{p}(\omega)\right|^{2} \operatorname{Re} \tilde{\sigma}_{\alpha \alpha}\left(T_{p}, \omega\right) .
\end{aligned}
$$

This result clearly indicates that the energy transfer from the probe pulse to electrons is described by the real part of the time-resolved conductivity that we defined by Eq. (27). 


\section{Parabolic Two-Band Model}

We introduce a two-band model in Eq. (21), considering only two orbitals in the sum, occupied valence $(v)$ and unoccupied conduction $(c)$ bands. The excitation energy from the valence band to the conduction band is assumed to have a parabolic form,

$$
\epsilon_{c \vec{k}}-\epsilon_{v \vec{k}} \simeq \frac{k^{2}}{2 \mu}+\epsilon_{g}
$$

where $\epsilon_{g}$ is the band gap energy and $\mu$ is the reduced mass of electron-hole pairs.

\section{Conductivity under a static electric field: static Franz-Keldysh effect}

For a static electric field $\vec{E}$, the vector potential has a linear time-dependence,

$$
\vec{A}_{p}(t)=\vec{A}_{p}(T)-c \vec{E}(t-T) .
$$

The conductivity $\sigma\left(t, t^{\prime}\right)$ of Eq. (21) is a function of $t-t^{\prime}$ and the conductivity $\sigma\left(T_{p}, \omega\right)$ of Eq. (25) is independent of $T_{p}$. After straightforward calculations, we have

$$
\begin{aligned}
& \tilde{\sigma}_{\alpha \beta}(\omega)=\frac{i e^{2} n_{e}}{m_{e} \omega} \delta_{\alpha \beta}+\frac{e^{2}}{m_{e}^{2} \omega V} \int_{0}^{\infty} d s e^{i \omega s} \sum_{\vec{k}} \\
& \times\left[\left(p_{\alpha}\right)_{v c \vec{k}-e \vec{E} s / 2}\left(p_{\beta}\right)_{c v \vec{k}+e \vec{E} s / 2} e^{-i\left\{\left(\epsilon_{k}+\epsilon_{g}\right) s+\frac{c^{2} E^{2}}{24 \mu} s^{3}\right\}}\right. \\
& \left.-\left(p_{\beta}\right)_{v c \vec{k}+e \vec{E} s / 2}\left(p_{\alpha}\right)_{c v \vec{k}-e \vec{E} s / 2} e^{i\left\{\left(\epsilon_{k}+\epsilon_{g}\right) s+\frac{c^{2} E^{2}}{24 \mu} s^{3}\right\}}\right]
\end{aligned}
$$

Below, we consider a real part of the diagonal element, $\operatorname{Re} \sigma_{\alpha \alpha}(\omega)$. We further assume that $\vec{k}$-dependence of the matrix elements $\left(p_{\alpha}\right)_{v c \vec{k}}$ may be ignored. Then, carrying out integration over $s$, we have

$$
\begin{aligned}
\operatorname{Re} \tilde{\sigma}_{\alpha \alpha}(\omega) & =\frac{\pi e^{2}}{m_{e}^{2} \omega V}\left|\left(p_{\alpha}\right)_{v c}\right|^{2} \sum_{\vec{k}}\left(\frac{8 \mu}{e^{2} E^{2}}\right)^{1 / 3} \\
& \times A i\left(\left(\epsilon_{k}+\epsilon_{g}-\omega\right)\left(\frac{8 \mu}{e^{2} E^{2}}\right)^{1 / 3}\right),
\end{aligned}
$$

where $A i(x)$ is the Airy function. Further carrying out $\vec{k}$-integration, we have

$$
\begin{aligned}
& \operatorname{Re} \tilde{\sigma}_{\alpha \alpha}(\omega)=\frac{(2 \mu)^{3 / 2} e^{2}}{2 m_{e}^{2} \omega}\left|\left(p_{\alpha}\right)_{v c}\right|^{2} \sqrt{\Theta} \\
\times & \left\{-\frac{\epsilon_{g}-\omega}{\Theta} A i^{2}\left(\frac{\epsilon_{g}-\omega}{\Theta}\right)+A i^{\prime 2}\left(\frac{\epsilon_{g}-\omega}{\Theta}\right)\right\},
\end{aligned}
$$

where $\Theta=\left(e^{2} E^{2} / 2 \mu\right)^{1 / 3}$. This is a well-known formula of static Franz-Keldysh effect [1].
2. Conductivity under a periodic electric field: dynamical Franz-Keldysh effect

We next consider a case of pump electric field which is periodic in time, $\vec{A}_{P}\left(t+T_{\Omega}\right)=\vec{A}_{P}(t)$, where $T_{\Omega}$ is the period of the pump field and is related to the frequency $\Omega$ by $T_{\Omega}=2 \pi / \Omega$. The conductivity $\sigma\left(t, t^{\prime}\right)$ has also the periodicity,

$$
\sigma\left(t, t^{\prime}\right)=\sigma\left(t-T_{\Omega}, t^{\prime}-T_{\Omega}\right) .
$$

We make a Fourier expansion of $\sigma_{\alpha \beta}(t, t-s)$ which is periodic in $t$ with the period $T_{\Omega}$,

$$
\sigma_{\alpha \beta}(t, t-s)=\sum_{n=-\infty}^{\infty} e^{i n \Omega t} \sigma_{\alpha \beta}^{(n)}(s)
$$

where $\sigma_{\alpha \beta}^{(n)}(s)$ is defined by

$$
\sigma_{\alpha \beta}^{(n)}(s)=\frac{1}{T_{\Omega}} \int_{0}^{T_{\Omega}} d t e^{-i n \Omega t} \sigma_{\alpha \beta}(t, t-s) .
$$

The time-resolved frequency-dependent conductivity $\tilde{\sigma}_{\alpha \beta}^{I}\left(T_{p}, \omega\right)$ for an impulsive probe field defined by Eq. (25) may be expressed as

$$
\tilde{\sigma}_{\alpha \beta}^{I}\left(T_{p}, \omega\right)=\sum_{n} e^{i n \Omega T_{p}} \tilde{\sigma}_{\alpha \beta}^{(n)}(\omega+n \Omega),
$$

where $\tilde{\sigma}_{\alpha \beta}^{(n)}(\omega)$ is the Fourier transform of $\sigma_{\alpha \beta}^{(n)}(s)$. For a general probe field of Eq. (26) with the envelope function $f_{p}(t)$, the conductivity defined by Eq. (27) becomes

$$
\begin{aligned}
\tilde{\sigma}_{\alpha \beta}\left(T_{p}, \omega\right) & =\frac{\int d s f(s) \tilde{\sigma}_{\alpha \beta}^{I}\left(T_{p}+s, \omega\right) e^{i\left(\omega-\omega_{0}\right) s}}{\int d s f(s) e^{i\left(\omega-\omega_{0}\right) s}} \\
& =\sum_{n} \frac{\tilde{f}_{p}\left(\omega+n \Omega-\omega_{0}\right)}{\tilde{f}_{p}\left(\omega-\omega_{0}\right)} e^{i n \Omega T} \sigma_{\alpha \beta}^{(n)}(\omega+n \Omega),
\end{aligned}
$$

where $\tilde{f}_{p}(\omega)$ is the Fourier transform of $f_{p}(t)$.

We now consider a specific form of the pump vector potential, $\vec{A}_{P}(t)=\vec{A}_{0} \cos \Omega t$, and calculate explicit form of the time-resolved conductivity assuming a two-band model. We calculate $\tilde{\sigma}_{\alpha \beta}^{(n)}(\omega)$ in two steps. We first calculate the Fourier transform of $\sigma_{\alpha \beta}(t, t-s)$ with respect to $s$ which we denote as $\hat{\sigma}_{\alpha \beta}(t, \omega)$. In the parabolic twoband model, it is given as follows.

$$
\begin{aligned}
& \hat{\sigma}_{\alpha \beta}(t, \omega)=\int_{-\infty}^{\infty} d s e^{i \omega s} \sigma_{\alpha \beta}(t, t-s) \\
= & \frac{i e^{2}}{m_{e} \omega} n_{e} \delta_{\alpha \beta}+\frac{e^{2}}{m_{e}^{2} \omega V} \int_{0}^{\infty} d s e^{i \omega s} \sum_{\vec{k}} \\
& {\left[\left(p_{\alpha}\right)_{v c}\left(p_{\beta}\right)_{c v} e^{-i \int_{0}^{s} d y\left\{\frac{1}{2 \mu}\left(\vec{k}+\frac{e}{c} \vec{A}(t-y)\right)^{2}+\epsilon_{g}\right\}}\right.} \\
& \left.-\left(p_{\beta}\right)_{v c}\left(p_{\alpha}\right)_{c v} e^{i \int_{0}^{s} d y\left\{\frac{1}{2 \mu}\left(\vec{k}+\frac{e}{c} \vec{A}(t-y)\right)^{2}+\epsilon_{g}\right\}}\right] .
\end{aligned}
$$


The integral in the exponential is calculated as

$$
\begin{aligned}
& \int_{0}^{s} d y\left\{\frac{1}{2 \mu}\left(\vec{k}+\frac{e}{c} \vec{A}(t-y)\right)^{2}+\epsilon_{g}\right\} \\
= & \left(\epsilon_{k}+\epsilon_{g}+U_{p}\right) s-\theta_{1} \sin \Omega(t-s)+\theta_{1} \sin \Omega t \\
& -\theta_{2} \sin 2 \Omega(t-s)+\theta_{2} \sin 2 \Omega t,
\end{aligned}
$$

where we introduced

$$
\begin{aligned}
& U_{p}=\frac{e^{2} A_{0}^{2}}{4 \mu c^{2}}, \\
& \theta_{1}=\frac{e \vec{k} \cdot \vec{A}_{0}}{\mu c \Omega}, \\
& \theta_{2}=\frac{e^{2} A_{0}^{2}}{8 \mu c^{2} \Omega} .
\end{aligned}
$$

Using a relation involving Bessel function $J_{n}(x)$,

$$
e^{i a \sin \theta}=\sum_{n} J_{n}(a) e^{i n \theta}
$$

where $n$ runs for whole integers, we may express

$$
\begin{aligned}
e^{i \theta_{1} \sin \Omega t+i \theta_{2} \sin 2 \Omega t} & =\sum_{l^{\prime} m} J_{l^{\prime}}\left(\theta_{1}\right) J_{m}\left(\theta_{2}\right) e^{i\left(l^{\prime}+2 m\right) \Omega t} \\
& =\sum_{l} J_{l}\left(\theta_{1}, \theta_{2}\right) e^{i l \Omega t}
\end{aligned}
$$

where we defined the generalized Bessen function by

$$
J_{l}\left(\theta_{1}, \theta_{2}\right)=\sum_{m} J_{l-2 m}\left(\theta_{1}\right) J_{m}\left(\theta_{2}\right) .
$$

We may express the integral in Eq. (42) as

$$
\begin{aligned}
& \int_{0}^{\infty} d s e^{i \omega s} \exp \left[-i\left[\left(\epsilon_{k}+\epsilon_{g}+U_{p}\right) s-\theta_{1} \sin \Omega(t-s)\right.\right. \\
& \left.\left.+\theta_{1} \sin \Omega t-\theta_{s} \sin 2 \Omega(t-s)+\theta_{2} 2 \Omega t\right]\right] \\
= & \sum_{l_{1} l_{2}} \frac{i J_{l_{1}}\left(\theta_{1}, \theta_{2}\right) J_{l_{2}}\left(\theta_{1}, \theta_{2}\right)}{\omega-\left(\epsilon_{k}+\epsilon_{g}+U_{p}+l_{1} \Omega\right)} e^{i\left(l_{1}-l_{2}\right) \Omega t} .
\end{aligned}
$$

Equation (42) is now written as

$$
\begin{aligned}
& \hat{\sigma}_{\alpha \beta}(t, \omega)=\frac{i e^{2}}{m_{e} \omega} n_{e} \delta_{\alpha \beta}+\frac{i e^{2}}{m_{e}^{2} \omega V} \sum_{\vec{k} l_{1} l_{2}} J_{l_{1}}\left(\theta_{1}, \theta_{2}\right) J_{l_{2}}\left(\theta_{1}, \theta_{2}\right) \\
& \times\left[\left(p_{\alpha}\right)_{v c}\left(p_{\beta}\right)_{c v} \frac{e^{i\left(l_{1}-l_{2}\right) \Omega t}}{\omega-\left(\epsilon_{k}+\epsilon_{g}+U_{p}+l_{1} \Omega\right)}\right. \\
& \left.-\left(p_{\beta}\right)_{v c}\left(p_{\alpha}\right)_{c v} \frac{e^{-i\left(l_{1}-l_{2}\right) \Omega t}}{\omega+\left(\epsilon_{k}+\epsilon_{g}+U_{p}+l_{1} \Omega\right)}\right] .
\end{aligned}
$$

We next calculate $\tilde{\sigma}_{\alpha \beta}^{(n)}(\omega)$ as follows.

$$
\begin{aligned}
& \tilde{\sigma}_{\alpha \beta}^{(n)}(\omega)=\frac{1}{T_{\Omega}} \int_{0}^{T_{\Omega}} d t e^{-i n \Omega t} \hat{\sigma}_{\alpha \beta}(t, \omega) \\
= & \frac{i e^{2}}{m_{e} \omega} n_{e} \delta_{\alpha \beta} \delta_{n 0}+\frac{i e^{2}}{m_{e}^{2} \omega V} \sum_{\vec{k} l} \\
& {\left[J_{l}\left(\theta_{1}, \theta_{2}\right) J_{l-n}\left(\theta_{1}, \theta_{2}\right) \frac{\left(p_{\alpha}\right)_{v c}\left(p_{\beta}\right)_{c v}}{\omega-\left(\epsilon_{k}+\epsilon_{g}+U_{p}+l \Omega\right)}\right.} \\
- & \left.J_{l}\left(\theta_{1}, \theta_{2}\right) J_{l+n}\left(\theta_{1}, \theta_{2}\right) \frac{\left(p_{\beta}\right)_{v c}\left(p_{\alpha}\right)_{c v}}{\omega+\left(\epsilon_{k}+\epsilon_{g}+U_{p}+l \Omega\right)}\right] .
\end{aligned}
$$

We put the above expression into Eq. (41),

$$
\begin{aligned}
\tilde{\sigma}_{\alpha \beta}\left(T_{p}, \omega\right)= & \sum_{n} \frac{\tilde{f}_{p}\left(\omega+n \Omega-\omega_{0}\right)}{\tilde{f}_{p}\left(\omega-\omega_{0}\right)} e^{i n \Omega T_{p}} \tilde{\sigma}_{\alpha \beta}^{(n)}(\omega+n \Omega) \\
= & \frac{i e^{2}}{m_{e} \omega} n_{e} \delta_{\alpha \beta} \\
+ & \sum_{\vec{k} l n} \frac{i e^{2}}{m_{e}^{2}(\omega+n \Omega) V} \frac{\tilde{f}_{p}\left(\omega+n \Omega-\omega_{0}\right)}{\tilde{f}_{p}\left(\omega-\omega_{0}\right)} e^{i n \Omega T_{p}} \\
\times & {\left[\frac{\left(p_{\alpha}\right)_{v c}\left(p_{\beta}\right)_{c v} J_{l}\left(\theta_{1}, \theta_{2}\right) J_{l-n}\left(\theta_{1}, \theta_{2}\right)}{\omega+n \Omega-\left(\epsilon_{k}+\epsilon_{g}+U_{p}+l \Omega\right)}\right.} \\
& \left.-\frac{\left(p_{\beta}\right)_{v c}\left(p_{\alpha}\right)_{c v} J_{l}\left(\theta_{1}, \theta_{2}\right) J_{l+n}\left(\theta_{1}, \theta_{2}\right)}{\omega+n \Omega+\left(\epsilon_{k}+\epsilon_{g}+U_{p}+l \Omega\right)}\right] .
\end{aligned}
$$

We carry out $\vec{k}$ integration in terms of $\epsilon_{k}=k^{2} / 2 \mu$ and $\cos \theta_{k}$. After integration over $\cos \theta_{k}$, only even $n$ terms contribute. We introduce the following quantity,

$$
\xi_{l n}(k)=\int_{-1}^{1} d\left(\cos \theta_{k}\right) J_{l}\left(\theta_{1}, \theta_{2}\right) J_{l-n}\left(\theta_{1}, \theta_{2}\right),
$$

and we denote $n=2 m$ below. For the diagonal term $\alpha=\beta$, we have

$$
\begin{aligned}
& \tilde{\sigma}_{\alpha \alpha}\left(T_{p}, \omega\right)=\frac{i e^{2}}{m_{e} \omega} n_{e} \\
+ & \sum_{l m} \frac{i e^{2} \mu^{3 / 2}\left|\left(p_{\alpha}\right)_{v c}\right|^{2}}{\sqrt{2} \pi^{2} m_{e}^{2}(\omega+2 m \Omega)} \frac{\tilde{f}_{p}\left(\omega+2 m \Omega-\omega_{0}\right)}{\tilde{f}_{p}\left(\omega-\omega_{0}\right)} e^{i 2 m \Omega T_{p}} \\
\times & \int_{0}^{\infty} \sqrt{\epsilon_{k}} d \epsilon_{k}\left[\frac{\xi_{l, 2 m(k)}}{\omega+2 m \Omega-\left(\epsilon_{k}+\epsilon_{g}+U_{p}\right)-l \Omega}\right. \\
& \left.-\frac{\xi_{l,-2 m}(k)}{\omega+2 m \Omega+\left(\epsilon_{k}+\epsilon_{g}+U_{p}\right)+l \Omega}\right]
\end{aligned}
$$

As the final result of this subsection, we obtain the following expression for the real part of the conductivity,

$$
\begin{aligned}
& \operatorname{Re} \tilde{\sigma}_{\alpha \alpha}\left(T_{p}, \omega\right)=\sum_{m=-\infty}^{\infty} \frac{e^{2} \mu^{3 / 2}\left|\left(p_{\alpha}\right)_{v c}\right|^{2}}{\sqrt{2} \pi^{2} m_{e}^{2}(\omega+2 m \Omega)} \\
& \times \frac{\tilde{f}_{p}\left(\omega+2 m \Omega-\omega_{0}\right)}{\tilde{f}_{p}\left(\omega-\omega_{0}\right)}\left[C_{m}(\omega) \cos 2 m \Omega T_{p}\right. \\
& \left.+S_{m}(\omega) \sin 2 m \Omega T_{p}\right] .
\end{aligned}
$$


Coefficients $C_{m}(\omega)$ are given by

$$
\begin{aligned}
& C_{m}(\omega)=\pi \int_{0}^{\infty} \sqrt{\epsilon_{k}} d \epsilon_{k} \\
\times & \sum_{l}\left\{\xi_{l, 2 m}(k) \delta\left(\omega+2 m \Omega-\left(\epsilon_{k}+\epsilon_{g}+U_{p}+l \Omega\right)\right)\right. \\
& \left.-\xi_{l,-2 m}(k) \delta\left(\omega+2 m \Omega+\left(\epsilon_{k}+\epsilon_{g}+U_{p}+l \Omega\right)\right)\right\} \\
= & \sum_{l} \pi\left[\sqrt{\epsilon_{k}^{+}} \xi_{l, 2 m}\left(k^{+}\right)-\sqrt{\epsilon_{k}^{-}} \xi_{l,-2 m}\left(k^{-}\right)\right],
\end{aligned}
$$

where $k$ and $k^{ \pm}$are related to $\epsilon_{k}$ and $\epsilon_{k}^{ \pm}$by $k=\sqrt{2 \mu \epsilon_{k}}$. $\epsilon_{k}^{ \pm}$are defined by

$$
\epsilon_{k}^{ \pm}= \pm(\omega+2 m \Omega)-\left(\epsilon_{g}+U_{p}+l \Omega\right) .
$$

Coefficients $S_{m}(\omega)$ are given by

$$
\begin{gathered}
S_{m}(\omega)=-\int_{0}^{\infty} \sqrt{\epsilon_{k}} d \epsilon_{k} \\
\times \sum_{l}\left[\frac{\xi_{l, 2 m}(k)}{\omega+2 m \Omega-\left(\epsilon_{k}+\epsilon_{g}+U_{p}+l \Omega\right)}\right. \\
\left.+\frac{\xi_{l,-2 m}(k)}{\omega-2 m \Omega+\left(\epsilon_{k}+\epsilon_{g}+U_{p}+l \Omega\right)}\right] .
\end{gathered}
$$

We note that the terms with $m= \pm 1$ are responsible for the phase shift seen in Fig. 2

The above expression of the time-resolved conductivity depends on the choice of the envelope function $f_{p}(t)$ through $\tilde{f}_{p}(\omega)$. Modulations with a long period are influenced by $\tilde{f}_{p}(\omega)$ at small $\omega$, typically $\omega \simeq \pm 2 \Omega$. This indicates that the conductivity does not depend much on the detail of the probe pulse, if we employ a probe pulse which is much shorter than the period $T$ of the pump. In the TDDFT calculation shown in Fig. 2, we used a short enough probe pulse with $\tilde{f}_{p}( \pm 2 \Omega) / f_{p}(0)=0.96$ close to unity.

\section{Numerical results}

We numerically calculate the change of the dielectric function $\Delta \operatorname{Im}\left[\varepsilon\left(\omega, T_{p}\right)\right]$ using Eqs. (56) - (59)). In the calculations shown below, we simply put $\tilde{f}(\omega)=1$. This is equivalent to using an impulsive field for the probe, $E_{p}(t) \propto \delta\left(t-T_{p}\right)$.

Figure 3 (a)-(d) show the contour plot, where the horizontal axis is the phase defined by $\Omega T_{p}$. In Fig. 3 (e)(h), modulations averaged over time is shown by red-solid lines. Blue-dashed lines show results of static FKE: the time-averaged modulation using static formula, Eq. (36) where the parameter $\theta$ is evaluated using time-dependent electric field, $E(t)$. The parameters in the two-band model are set to reproduce responses in the TDDFT calculations: The frequency of pump field is set to $\Omega=0.4$ $\mathrm{eV}$, the reduced mass is set to $\mu=0.25 m_{e}$, and the
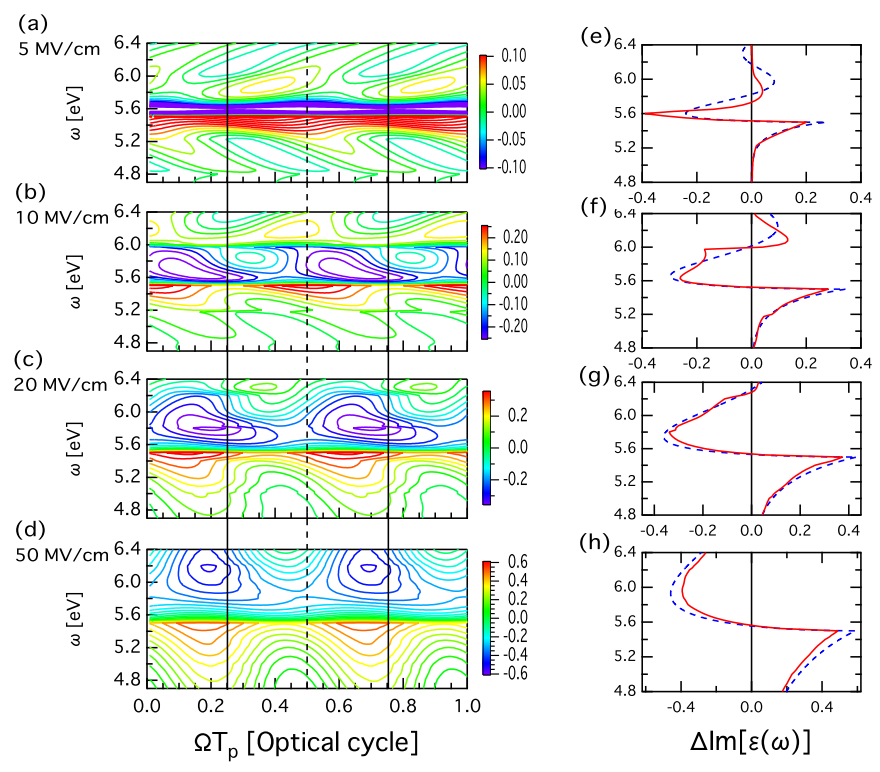

FIG. 3. Contour plots and time averages of $\Delta \operatorname{Im}\left[\varepsilon\left(\omega, T_{p}\right)\right]$ in the two-band model for the pump field intensities of $(\mathrm{a}, \mathrm{e}) E_{0}=$ 5, (b,f) 10, (c,g) 20, and (d,h) $50 \mathrm{MV} / \mathrm{cm}$. In the left panels (a)-(d), the horizon axis is the phase defined by $\Omega T_{p}$. The vertical solid (dashed) lines indicate the position of maximum (minimum) of the electric field. In the right panels (e)-(h), red-solid lines is the time average of the modulation. Bluedotted lines show result of static FKE.

dipole matrix element is set to $\left|p_{c v}\right|^{2}=0.928$ in atomic unit.

We first look at time-averaged modulations shown in panels (e)-(h). In all panels, we find appearances of absorption below the band gap and decrease above the band gap. We can also find structures of at $\omega=\epsilon_{g} \pm \Omega$, most clearly in (b) and (f), which are cause by side band contributions with different $l$ values in Eqs. (57) and (59). They show a similar behavior to Fig. 2 (g)-(k). We also find that the time-averaged modulation is quite close to the estimation by the static FKE, even at the smallest intensity where the $\gamma$ value is much less than unity. We repeat a similar calculation changing the frequency of the pump field while the intensity is fixed at $50 \mathrm{MV} / \mathrm{cm}$, and have found that the static FKE describes reasonably the time-averaged modulations below the band gap.

We next look at the time dependence of the modulation. At the strong pump electric field (d), we find a large modulation when the magnitude of the pump electric field is large. We find a phase shift as the pump electric field decreases. The maximum of the modulation moves forward in time. From these observations, we can say that all the features seen in the first-principles TDDFT calculations shown in Fig. 2 are reproduced by the analytic formula of Eqs. (56) - (59) of the simple two-band model. 


\section{SUMMARY}

We have developed a theoretical pump-probe formalism to investigate the dynamical Franz-Keldysh effect in time-domain, in femtosecond time resolution much shorter than the optical cycle of the applied pump field. Both numerical simulations based on real-time timedependent density functional theory and an analytic approach in the two-band model reveal the same behavior for the modulations of dielectric properties. We find the time-averaged behavior in the DFKE can be well described by the static FKE. The most remarkable feature of the DFKE is that there appears a phase shift between the modulation of the dielectric function and the applied pump field which becomes significant as the magnitude of the electric field decreases.

\section{ACKNOWLEDGMENTS}

The authors thank G.F. Bertsch for discussions and for carefully reading manuscript. This work is supported by a Grant-in-Aid for Scientific Research (No. 21740303 and No. 15H03674). Numerical calculations were performed on the supercomputer PRIMARGY BX900 at the Japan Atomic Energy Agency (JAEA) and the K computer at the RIKEN Advanced Institute for Computational Science (proposal number hp120065).
[1] M. Hentschel, R. Klenberger, Ch. Spielmann, G.A. Reider, N. Milosevic, T. Brabec, U. Heinzmann, M. Drescher, and F. Krausz, Nature 414, 509 (2001).

[2] H. Hirori, A. Doi, F. Blanchard, and K. Tanaka, Appli. Phys. Lett. 98, 091106 (2011).

[3] A.H. Chin, O.G. Calderon, and J. Kono, Phys.Rev. Lett. $\mathbf{8 6}, 3292$ (2001).

[4] H.Hirori, K. Shinokita, M. Shirai, S. Tani, Y. Kadoya, and K. Tanaka, Nat. Comm. 2, 594 (2011).

[5] A. Schiffrin, T. Paasch-Colberg, N. Karpowicz, V. Apalkov, D. Gerster, S. Mühlbrandt, M. Korbman, J. Reichert, M. Schultze, S. Holzner, J.V. Barth, R. Kienberger, R. Ernstorfer, V.S. Yakovlev, M.I. Stockman, and F. Krausz, Nature 493, 70 (2013).

[6] M. Schultze, E.M. Botschafter, A. Sommer, S. Holzner, W. Schweinberger, M. Fless, M. Hofstetter, R. Kienberger, V. Apalkov, V.S. Yakovlev, M.I. Stockman, F. Krausz, Nature 493, 75 (2013).

[7] F. Nobelli, D. Fausti, F. Giusti, F. Parmigiani, and M. Hoffmann, Scientific Reports 31227 (2013).

[8] M. Schultze, K. Ramasesha, C.D. Pemmaraju, S.A. Sato, D. Whitmore, A. Gandman, J.S. Prell, L.J. Borja, D. Prendergast, K. Yabana, D.M. Neumark, S.R. Leone, Science 346, 1348 (2014).

[9] W.Franz, Z. Naturforsch. Teil A 13, 484 (1958).

[10] L. V. Keldysh, Sov. Phys. JETP 34, 788 (1958).

[11] K. Tharmalingam, Phys. Rev. 130, 2204 (1963).

[12] B. O. Seraphin and R. B. Hess, Phys. Rev. Lett. 14, 138 (1965).

[13] R. E. Nahory and J. L. Shay, Phys. Rev. Lett. 21, 1569 (1968).

[14] H.Shen, M. Dutta, J. Appl. Phys. 78, 2151 (1995).

[15] J.K. Wahlstrand, J.E. Sipe, Phys. Rev. B82, 075206 (2010).

[16] F. Duque-Gomez, J.E. Sipe, J. Phys. Chem. Solids 76, 138 (2015).

[17] Y. Yacoby, Phys. Rev. 169, 610 (1968).

[18] A. P. Jauho and K. Johnsen, Phys.Rev. Lett. 76, 4576 (1996).

[19] K. B. Nordstrom, K. Johnsen, S. J. Allen, A. P. Jauho, B. Birnir, J. Kono, T. Noda, H. Akiyama, and H. Sakaki, Phys. Rev. Lett. 81, 457 (1998).
[20] Ajit Srivastava, Rahul Srivastava, Jigang Wang, and Junichiro Kono, Phys.Rev.Lett. 93, 157401 (2004).

[21] Y. Mizumoto, Y. Kayanuma, A. Srivastava, J. Kono, and A. H. Chin, Phys. Rev. B 74, 045216 (2006).

[22] S. Ghimire, A.D. DiChiara, E. Sistrunk, U.B. Szafruga, P. Agostini, L. F. DiMauro, and D. A. Reis, Phys. Rev. Lett. 107, 167407 (2011).

[23] A. H. Chin, J. M. Bakker, and J. Kono, Phys. Rev. Lett. 85, 3293 (2000).

[24] Ajit Srivastava, Rahul Srivastava, JigangWang, and Junichiro Kono, Phys. Rev. Lett. 93, 157401 (2004).

[25] E. Runge and E. K. U. Gross, Phys.Rev. Lett. 52, 997 (1984).

[26] G.F. Bertsch, J.-I. Iwata, A. Rubio, and K. Yabana, Phys. Rev. B 62, 7998 (2000).

[27] N. Troullier and J.L. Martins, Phys. Rev. B43, 1993 (1991).

[28] T. Otobe, M. Yamagiwa, J. -I. Iwata, K. Yabana, T. Nakatsukasa, and G. F. Bertsch, Phys. Rev. B77, 165104 (2008).

[29] T. Otobe, K. Yabana, J.-I. Iwata, J. Phys.: Condens. Matter. 21, 064224 (2009).

[30] Y. Shinohara, K. Yabana, Y. Kawashita, J.-I. Iwata, T. Otobe, and George F. Bertsch, Phys. Rev. B 82, 155110 (2010).

[31] Y. Shinohara, S. A. Sato, K. Yabana, J.-I. Iwata, and T. Otobe, J. Chem. Phys. 137 22A527 (2012).

[32] T. Otobe, J. Appli. Phys. 111, 093112 (2012).

[33] S.A. Sato, K. Yabana, Y. Shinohara, T. Otobe, G.F. Bertsch, Phys. Rev. B89, 064304 (2014).

[34] G. Wachter, C. Lemell, J. Burgdorfer, S.A. Sato, X.M. Tong, K. Yabana, Phys. Rev. Lett. 113, 087401 (2014).

[35] N. Troullier and J.L. Martins, Phys. Rev. B43, 1993 (1991).

[36] L. Kleinman and D. M. Bylander, Phys. Rev. Lett. 48, 1425 (1982).

[37] J. P. Perdew and A. Zunger, Phys. Rev. B 23, 5048 (1981).

[38] K. Yabana and G.F. Bertsch, Phys. Rev. B54, 4484 (1996).

[39] K. Yabana, T. Sugiyama, Y. Shinohara, T. Otobe, and G. F. Bertsch, Phys. Rev. B 85, 045134 (2012). 
[40] G. Vignale and W. Kohn, Phys. Rev. Lett. 77, 2037 (1996).

[42] W. V. Houston, Phys. Rev. 51, 184 (1940).

[41] K. Yabana, T. Nakatsukasa, J.-I. Iwata, and G. F. Bertsch, Phys. Stat. Solidi (b) 243, 1121 (2006) 